This is a peer-reviewed paper published in Minerals Engineering.

https://doi.org/10.1016/j.mineng.2021.107054

Lucas Pereira*a, Max Frenzel a, Duong Huu Hoanga,b, Raimon Tolosana-Delgado ${ }^{a}$, Martin Rudolph ${ }^{a}$, Jens Gutzmer ${ }^{a}$

a. Helmholtz-Zentrum Dresden-Rossendorf, Helmholtz Institute Freiberg for Resource Technology, Chemnitzer Straße 40, 09599 Freiberg, Germany

b. Maelgwyn Mineral Services Ltd, Ty Maelgwyn, 1A Gower Road, Cathays, Cardiff, CF24 4PA, United Kingdom

*Corresponding author: I.pereira@hzdr.de 


\title{
Computing single-particle flotation kinetics using automated mineralogy data and machine learning
}

Lucas Pereira $^{\mathrm{a}^{*}}$, Max Frenzel ${ }^{\mathrm{a}}$, Duong Huu Hoang ${ }^{\mathrm{a}, \mathrm{b}}$, Raimon Tolosana-Delgado ${ }^{\mathrm{a}}$, Martin Rudolph ${ }^{\mathrm{a}}$, Jens Gutzmer ${ }^{\mathrm{a}}$

a Helmholtz-Zentrum Dresden-Rossendorf, Helmholtz Institute Freiberg for Resource Technology, Chemnitzer Straße 40, 09599 Freiberg, Germany

${ }^{b}$ Maelgwyn Mineral Services Ltd, Ty Maelgwyn, 1 A Gower Road, Cathays, Cardiff, CF24 4PA, United Kingdom

*Corresponding author: I.pereira@hzdr.de

\begin{abstract}
Studies of flotation kinetics are essential for understanding, predicting, and optimizing the selective recovery of minerals and metals through flotation. Recently, much effort has been made to use intrinsic ore properties to model flotation behavior. Particle-based characterization methods, e.g. SEM-based image analysis, has enabled much of this development. However, currently available methods for studies of flotation kinetics can not accommodate single-particle data, resulting in incomplete use of data that is readily available today. In this contribution, a method is introduced to apply kinetic flotation models to individual particles. This method, based on lasso-regularized multinomial logistic regression, allows for an in-depth understanding of particle flotation behavior as a function of all measured particle characteristics. With the proposed method, the joint influences of particle size, shape, as well as modal and surface compositions on the recovery of individual particles can be taken into unprecedented consideration. The results of the simulated particle behavior showed a very good agreement to the outcome of conventional empirical studies and follow well-described froth flotation recovery behavior.
\end{abstract}

Keywords: Geometallurgy; process mineralogy; machine learning; froth flotation; particle-based separation modelling

\section{Introduction}

Scanning electron microscope (SEM)-based automated mineralogy systems such as the Mineral Liberation Analyzer (MLA) or TIMA-X have revolutionized the fields of process mineralogy and geometallurgy by providing particle-based characterization data at unprecedented resolution (Fandrich et al., 2007). Their development and application continues to have considerable impact on the mining industry by improving the understanding of mineral separation processes, and thus reducing technical as well as environmental risks (Gu et al., 2014). This is particularly true for complex ores (Frenzel et al., 2019; Kern et al., 2018; Lamberg and Vianna, 2007).

Despite its successful track record, the technique still holds considerable potential to improve process understanding. This is so, because current best-practice process modelling tools are based on fitting particle distribution models (King et al., 2012), using only part of the available particle datasets. This is especially true for flotation, which is the most widely used mineral separation process. The number of microprocesses in 
This is a peer-reviewed paper published in Minerals Engineering. Please submit any inquiries to the corresponding author. https://doi.org/10.1016/j.mineng.2021.107054

flotation and their interactions hinders the development of process models that are capable of capturing most of the inherent complexity (King et al., 2012). The current use of particle data in the modelling of flotation kinetics is also still insufficient to understand the full complexity of the process. This is so, because much critical information contained in particle datasets is lost during the extensive data compression steps necessary for the application of current methods (e.g. particle grouping/binning and variable selection) (Gorain et al., 2000; Hoang et al., 2018; Jameson, 2012; Lamberg and Vianna, 2007; Lotter et al., 2011).

Most successful flotation models consider froth flotation as a kinetic process, in analogy to chemical reactions (Dowling et al., 1985; King et al., 2012; Sutherland, 1948). In these models, the floatable mineral particles and bubbles are analogous to the reactants, and the mineral particles attached to the bubbles are the product. The transfer rate of particles from the pulp to the concentrate is a function of the probabilities of collision, attachment, and detachment of particles with, to and from bubbles as well as their recovery through the froth zone (King et al., 2012).

Even though mathematical functions exist to quantitatively describe each of these probabilities at the micro-scale (Albijanic et al., 2010; Do, 2010; King et al., 2012; Wang et al., 2015; Yoon and Mao, 1996; Yoon et al., 2012), their complexity and the number of free parameters they contain hold back their extensive application in flotation modelling (Polat and Chander, 2000). Therefore, simplified models describing particle recovery on the macro-scale while still considering flotation as a kinetic process are generally applied in practice (King et al., 2012; Polat and Chander, 2000). These models successfully capture the nature of the flotation process in a well-stirred flotation environment. A multitude of such simplified kinetic flotation models are available in the literature (Gharai and Venugopal, 2015; Polat and Chander, 2000). These models are obtained by solving the differential equation describing a kinetic process (e.g. Eq. (1), Polat and Chander, 2000), where $C$ and $C_{b}$ are the concentrations of particles and bubbles, respectively, the exponents $n$ and $m$ are the reaction orders, $t$ is time and $k$ is the flotation rate constant.

$$
\frac{d C}{d t}=-k_{n} C^{n} C_{b}^{m}
$$

With regards to the material being processed, most of the differences between available models reflect variations in the assumed distributions of mineral composition, mineral hydrophobicity and particle size (Polat and Chander, 2000). As demonstrated by Polat and Chander (2000), very good predictive accuracy can be achieved with simple flotation models when subdividing ore particles into classes of similar behavior. Thus, the classical first-order flotation rate constant model (Eq. (2), Sutherland, 1948) is one of the most widely used flotation models (q.v. Section 2.3). It models $R_{t}$, the recovery at time $t$, as a function of $R_{\max }$, the maximum recovery of a mineral at $t=\infty$, and its rate constant $k$ :

$$
R_{t}=R_{\max }\left(1-e^{-k t}\right)
$$

To fit this kind of model to the behavior of a certain chemical element, mineral or particle class in a process, recovery needs to be determined as a function of time. This can be achieved with laboratory flotation experiments, or with a specific sampling campaign in industrial operations, designed to collect concentrate fractions corresponding to different flotation times (Runge, 2010; Wills and Finch, 2015). In the case of industrial operations, 
This is a peer-reviewed paper published in Minerals Engineering. Please submit any inquiries to the corresponding author. https://doi.org/10.1016/j.mineng.2021.107054

continuous models of flotation kinetics should be considered (Wills and Finch, 2015). Subsequently, the recovery of component $a$ in the concentrate at time $t, R_{a}^{t}$, can be calculated (Eq. (3)) based on the grade of component $a$ in the concentrate at time $t, G a_{c}^{t}$, and in the original feed, $G a_{f}$, as well as the masses of these two fractions, $M_{c}^{t}$ and $M_{f}$

$$
R_{a}^{t}=\frac{M_{c}^{t} \cdot G a_{c}^{t}}{M_{f} \cdot G a_{f}}
$$

Once recovery as a function of time has been determined, the $k$ and $R_{\max }$ parameters for each of the components can be obtained by fitting kinetic flotation models using nonlinear least squares regression. The goodness of fit for a fit kinetic flotation model can be evaluated using the corresponding residuals (Wills and Finch, 2015).

To obtain the grades of chemical elements or minerals in the different materials, standard chemical (e.g X-Ray fluorescence spectometry, Jenkins, 2008) or mineralogical (e.g. Xray powder diffraction, Bish and Post, 1989) bulk assays are usually sufficient. Yet, the routine application of bulk resource characterization methods has been largely superseded by the possibilities to obtain particle-level information. For this purpose, several techniques are now widely available. The most comprehensive particle-level data is currently provided by SEM-based image analysis (aka automated mineralogy). This technique is capable of characterizing the modal mineralogy, surface composition, size, and shape of individual particles in a sample (Fandrich et al., 2007), albeit with certain shortcomings due to stereologic degradation effects (Miller et al., 2009). However, particles characterized in one sample cannot be directly matched to the particles characterized in other samples - i.e. particles observed on the feed cannot be matched with particles from the concentrate or the tailings. Thus, approaches have been developed in which particles with similar characteristics are binned into a finite numbers of classes (e.g. via thresholds for free surface, particle size, or mineral association). Because these classes are present in all samples, they can then be used to calculate recoveries according to Eq. (3) and fit standard kinetic flotation models (Gorain et al., 2000; Hoang et al., 2018; Jameson, 2012; Lamberg and Vianna, 2007; Lotter et al., 2011).

Extending this previous work, Pereira et al. (2021) recently proposed a novel particlebased separation model (PSM) that allows for the estimation of recovery probabilities for individual particles. The close link between such probabilities and bulk recoveries (Jowett, 1986; King et al., 2012; Tromp, 1937) provides the unique opportunity to understand particle flotation kinetics at the single-particle level. In this contribution we present a PSM for the analysis of the kinetic behavior at the particle level. This builds upon an extension of Pereira et al (2021) in order to deal with more than two output streams. This allows us to compute the cumulative recovery probability of individual particles during flotation test-work as a function of time, which can then be fit by kinetic flotation models. The result provides insights into the flotation process at an unprecendented level of detail.

A detailed description of our method is provided in Section 2 below. Its application is illustrated using real data from the flotation test work of Hoang et al. (2018) in Section 3. Finally, Section 4 presents a general discussion of the results together with an outlook on the potential impact of the method. 
This is a peer-reviewed paper published in Minerals Engineering. Please submit any inquiries to the corresponding author. https://doi.org/10.1016/j.mineng.2021.107054

\section{Method}

The following subsections provide a brief outline of the method used in this manuscript to fit kinetic flotation models to single particles. The method can be used at any scale, in laboratory- or pilot-scale testing, as well as in industrial operations.

First, no distinct flotation testing procedure is required, yet a brief overview on data acquisition is presented in Section 2.1. Following this, the machine learning-based strategy to obtain the cumulative recovery probability of each particle in the feed sample as a function of flotation time is presented in Section 2.2. Lastly, Section 2.3 highlights important aspects of the different kinetic flotation models available, identifying the most suitable model to be used for particles. Figure 1 illustrates the overall workflow.

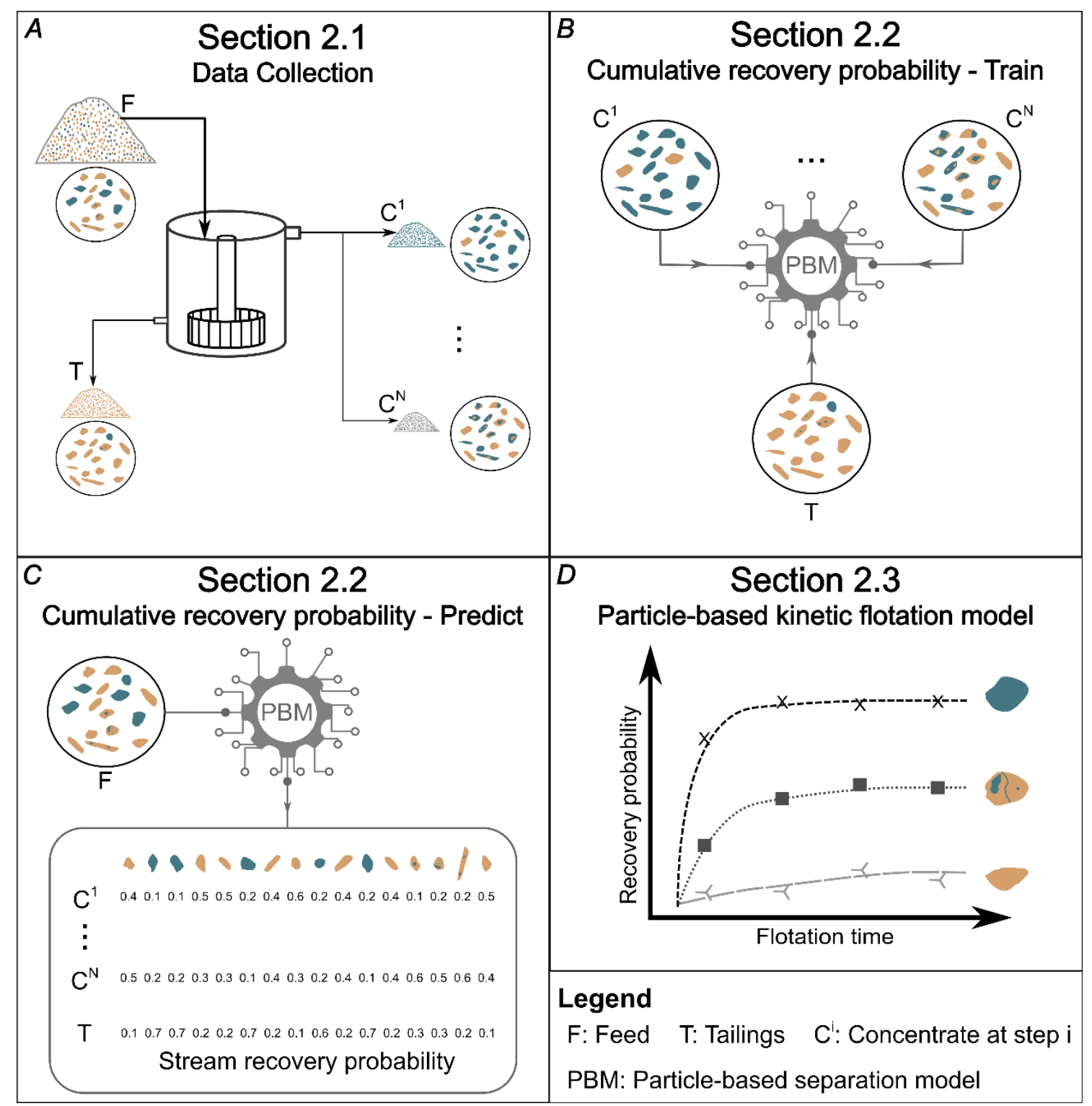

Figure 1: Schematic representation of overall workflow. First, the data is collected according to the procedure(s) described in section 2.1 (A). Using data from the output stream, particle-based separation models are trained $(B)$. The recovery probability for each particle and stream is then calculated for the original feed sample (C). Finally, a kinectic flotation model is fit to these particles (D). 
This is a peer-reviewed paper published in Minerals Engineering. Please submit any inquiries to the corresponding author. https://doi.org/10.1016/j.mineng.2021.107054

\subsection{Data collection}

Similar sampling strategies are usually followed during studies of flotation kinetics at the laboratory, pilot, or industrial scale: one must obtain different concentrate samples that represent well-defined intervals of flotation time, in addition to the feed and final tailings (Figure $1 \mathrm{~A}$ ). In our method, we require one sample from each concentrate, one sample of the final tailings, and one sample of the feed (q.v. Section 2.2). The feed sample is then used for validation (q.v. Section 2.2).

In a standard laboratory batch test, sample collection is done by exchanging the froth collection tray after defined time intervals, while in the pilot or industrial scale, it is done by collecting samples of the concentrate fraction from different flotation cells or banks, representing different residence times of the particles (Runge, 2010; Wills and Finch, 2015). For more details on flotation test procedures, the reader is referred to dedicated literature on the topic (Dobby and Savassi, 2005; Runge, 2010; Wills and Finch, 2015).

In the next step, particles in the collected samples need to be characterized according to their mineralogical composition, size, and shape. Any characterization technique that delivers such information can be used. SEM-based image analysis using the mineral liberation analyzer (MLA) software is used in this study. Recommendations for sample preparation and analysis in recognized scientific literature should be followed in the acquisition of this data (Fandrich et al., 2007; Heinig et al., 2015; Sandmann, 2015).

After characterization, the collected particle datasets are ready to be processed virtually. Due to the amount of data to be processed $(\sim 150,000$ particles per sample, each described by $\sim 70$ variables), the use of a programming environment is required. This study follows the internal procedure of the Helmholtz Institute Freiberg for Resource Technology (HIF) to handle large particle datasets in $R$ ( $R$ Core Team, 2017), as introduced by Kupka et al. (2020).

After importing the particle datasets, the variables describing particle properties need to be pre-treated before further processing (Pereira et al., 2021), as follows:

- Closure (Pawlowsky-Glahn et al., 2015) of the modal mineralogy,

- Closure of the surface composition,

- Log-transformation of the equivalent circle diameter $(E C D)$, solidity, and aspect ratio,

- Addition of the square of the log-transformed $E C D$ as an extra variable $\left(E C D^{2}\right)$.

- Addition of a categorical variable indicating the main mineral (by mass) of each particle (mainmineral).

\subsection{Cumulative recovery probability}

To estimate cumulative recovery probabilities for each particle during flotation test work, we use a modified version of the method presented in Pereira et al. (2021). While the original version of this method was introduced for separation processes producing only two output streams, we introduce a generalized version here that is able to deal with processes with any number of output streams.

The machine learning-based PSM from Pereira et al. (2021) consists of training a least absolute shrinkage and selection operator (lasso)-regularized logistic regression model (Hastie et al., 2015) using a particle dataset from each process product obtained. In addition, it uses a prior probability adjustment step (Saerens et al., 2002) to accommodate geological variability (Hoal, 2008). In this contribution, the lassoregularized logistic regression is substituted by a lasso-regularized multinomial logistic 
This is a peer-reviewed paper published in Minerals Engineering. Please submit any inquiries to the corresponding author. https://doi.org/10.1016/j.mineng.2021.107054

regression (Hastie et al., 2015). This substitution allows for the accommodation of multiple classes (i.e. process products) while still being self-adaptive (i.e. no human input required) and able to accommodate geological variability.

To obtain the training data for the multinomial logistic regression, a single data frame containing the pre-treated particle data from each concentrate stream and the final tailings needs to be compiled. In order to achieve, on average, equal representation of each particle class in the training data (cf. Pereira et al., 2021), a statistical weight is assigned to each particle according to its class. The statistical weight $\left(S W_{p . s j}\right)$ of each particle in sample $j$ is described by the following formula:

$$
S W_{p . s j}=\frac{1}{N P_{s j}}
$$

Where $N P_{s j}$ is the number of particles in particle dataset $j$. If more than one particle dataset is available for each class (e.g. if the sample is split into different size fractions before analysis), a more detailed approach for constructing the training dataset is required - this topic is discussed in the Supplementary Material 1.

After compiling the training dataset, the lasso-regularized multinomial logistic regression PSM is trained (Figure $1 \mathrm{~B}$ ) using an analysis of covariance structure (Keppel and Wickens, 2004), which contains interaction terms between shape and size variables and the mainmineralvariable. This model structure is used to capture a mineral-specific effect of particle dimension properties on recoverability (Pereira et al., 2021).

The quality of each PSM should be evaluated before proceeding to the next steps. This procedure is explained in detail by Pereira et al. (2021). It consists of using bootstrapping (Henderson, 2005) to virtually simulate the mineral separation process multiple times. Resampling of the feed particle population (same number of particles, with replacement) is also done to evaluate sampling uncertainty. The distribution of grades and masses obtained for each process product from the bootstrapping, are compared to the actual ones obtained in the test using a boxplot representation of natural log-ratios. Ideally, they should not deviate significantly from 0 (eqv. to real ratio of 1 ), indicating that sampling and modelling are reliable.

Finally, we use the trained particle-based separation model to compute the probabilities of each particle in the feed sample to be recovered in each of the different concentrate fractions, or to report to the tailings (Figure $1 \mathrm{C}$ ). The cumulative sum of the probability of a particle to be recovered in the concentrate fractions over flotation time is the cumulative recovery probability. With this information, we can fit kinetic flotation models to each particle as detailed in Section 2.3.

\subsection{Particle-based kinetic flotation model}

The last step of our proposed method consists of fitting the classical first-order kinetic flotation model (Eq. (2), Sutherland, 1948) to each particle using non-linear least squares regression. Following, we justify the use of this specific kinetic flotation model with a brief explanation of the relationship between recovery probability for individual particles, and overall recovery for a specific component or particle bin in a flotation test.

According to the general description of flotation processes given by King et al. (2012), each particle must collide, adhere, and not detach from a rising bubble during the pulp, pulp-froth interface, and froth phases in order to be recovered. The mean rate of occurrence of these processes can be calculated for particle bins (i.e. groups of particles with similar composition and physical properties) using their recovery (Eq. (3)). Yet not 
This is a peer-reviewed paper published in Minerals Engineering. Please submit any inquiries to the corresponding author. https://doi.org/10.1016/j.mineng.2021.107054

every occurrence of these processes is successful, and given the complex nature of the microprocesses governing their chance of success, it is best to use probabilities to quantify their efficiency (King et al., 2012). The recovery of a particle bin, which generally contains a large number of individual particles, is therefore equivalent to the average recovery probability of the particles in that bin. Thus, the use of recovery probabilities for the kinetic flotation modelling of individual particles represents the logical step forward to a higher resolution in process understanding and modelling (cf. Pereira et al., 2020). This is what we have done in this study. These considerations also show that recovery probability and recovery are essentially equivalent, the major difference being the number of particles they describe.

As a further note, the probabilities used in this work describe the success of the macro flotation process, without breaking it up into collision, attachment, and detachment. This is done, because sampling to describe the individual micro-processes levels is generally not possible. The increase in resolution offered by our method is therefore due to use of the single-particle data rather than particle bins.

Based on the previously calculated cumulative recovery probabilities, a flotation model is fitted to each particle with non-linear least squares regression (Figure $1 \mathrm{D}$ ). The discussion about the singularities of the available first-order flotation models started in Section 1 is extended here to identify the most suitable kinetic flotation model for single particles. We limit our discussion here to first-order flotation models for these being the most used in practice. Extensive literature is available for higher order models (Gharai and Venugopal, 2015; King et al., 2012; Polat and Chander, 2000).

The main difference between available first-order flotation models is their flotation rate constant distribution function (Dowling et al., 1985; Polat and Chander, 2000). Dowling et al. (1985) advocated that no single distribution function can be named "best", but the most suitable function has to be identified for each case. This is caused by the singularities in the formation of different mineral deposits (Guilbert and Park, 2007), which results in different intrinsic ore properties (e.g. mineralogy, grain sizes, microstructure or texture, and intergrowth relationships) that are later translated into distinct distribution functions of particle size and hydrophobicity - factors controlling the flotation rate distribution function.

On the other hand, simple flotation models can deliver high predictive accuracy if the ore is subdivided into classes of similar behavior (Polat and Chander, 2000). That is because fitting distribution functions to intrinsic particle properties is generally difficult due to the non-trivial distributions of these properties: zero-inflated, skewed, and multimodal (Pereira et al., 2021).

In our method, we capture the individual behavior of each characterized particle, which might be understood as using the maximum number of particle bins possible, thus justifying the use of the classical first-order model (Eq. (2), Sutherland, 1948) - the kinetic flotation model with the most simple flotation rate constant distribution.

\section{Demonstration}

We demonstrate the capability of our method using the data from the case study of Hoang et al. (2018). First, this dataset is briefly introduced in Section 3.1. Following, we validate the quality of the particle-based separation models trained on this dataset in Section 3.2.1, and of the kinetic flotation models fit to each particle in Section 3.2.2. Finally, we use the obtained results to understand the influence of selected particle 
This is a peer-reviewed paper published in Minerals Engineering. Please submit any inquiries to the corresponding author. https://doi.org/10.1016/j.mineng.2021.107054

properties on flotation behavior in Section 3.2.3. For this analysis, we calculate the modified flotation rate constant $\left(k_{m}\right)$ introduced by $\mathrm{Xu}$ (1998). This constant corresponds to the product of $k$ and $R_{\max }$ (cf. Eq. (2)); it represents the slope of the tangent of the recovery-time curve at zero time, and is used since some operating conditions can affect $R_{\text {max }}$ but not $k$, and vice-versa. Thus, $k_{m}$ provides a better insight on the influence of each particle property (e.g. mineral composition, liberation, size, shape, etc.) on a particle's overall flotation behavior.

\subsection{Materials and methods}

For the study of Hoang et al. (2018), phosphate rock samples from the Lao Cai province, Vietnam, were provided by the Vietnam Apatite Limited Company. The objective of the flotation experiments was to determine the best way to efficiently separate fluorapatite from dolomite, calcite and silicates. The sampling and crushing procedures used are described in Leißner et al. (2016).

Table 1 shows the modal mineralogy of the feed sample. Twenty three minerals were identified with the MLA. They are grouped into apatite, dolomite, calcite, micas, other silicates, sulfides, and oxides in the following sections, figures and tables for better visualization. Phlogopite, quartz, pyrite, and hematite, respectively, are the major minerals in the multi-mineralic groups. The apatite grade of the ore is high (64.1 wt.\%), and dolomite is the main gangue mineral (20.7 wt.\%).

Table 1: Grouped modal mineralogy of the feed sample, obtained with the MLA

\begin{tabular}{c|ccccccc} 
& Apatite & Dolomite & Calcite & $\begin{array}{c}\text { Other } \\
\text { silicates }\end{array}$ & Micas & Sulfides & Oxides \\
\hline $\begin{array}{c}\text { Feed } \\
\text { (wt.\%) }\end{array}$ & 64.1 & 20.7 & 6.1 & 5.6 & 2.4 & 1.1 & 0.1
\end{tabular}

After grinding for 8 minutes in a laboratory ball mill to assure a d90 of $67 \mu \mathrm{m}$, batch flotation tests were performed in a flotation cell built at the TU Bergakademie Freiberg (Figure $1 \mathrm{~A})$. Corn starch $\left(\left(\mathrm{C}_{6} \mathrm{H}_{10} \mathrm{O}_{5}\right)_{n}\right)$ gelatinized with sodium hydroxide $(\mathrm{NaOH})$ was used in combination with sodium silicate $\left(\mathrm{Na}_{2} \mathrm{SiO}_{3}\right)$ to depress gangue minerals. The latter also acts as a fine particle dispersant. Solution $\mathrm{pH}$ was kept at 10 using the modifier sodium carbonate $\left(\mathrm{Na}_{2} \mathrm{CO}_{3}\right)$, which can also be regarded as a depressant. Berol 2015 was used as the collector. Four concentrate fractions were collected after $0.75 \mathrm{~min}$ (CA), $1.50 \mathrm{~min}(\mathrm{CB}), 3.00 \mathrm{~min}(\mathrm{CC})$, and $6.00 \mathrm{~min}(\mathrm{CD})$. In addition, a final tailings sample was collected (TD). Five replicates of the test were done to ensure reproducibility and produce enough sample mass for detailed characterization.

All samples, including the feed, were wet sieved into four size fractions $(-20 \mu \mathrm{m},+20$ to $-32 \mu \mathrm{m},+32$ to $-50 \mu \mathrm{m}$, and $+50 \mu \mathrm{m}$ ) before characterization by MLA at the Helmholtz Institute Freiberg for Resource Technology. Samples were prepared following the procedure described by Heinig et al. (2015), and analyzed on a FEI Quanta 650F scanning electron microscope equipped with two Bruker Quantax X-Flash 5030 EDX detectors. The SEM was operated at $25 \mathrm{kV}$ overall electron beam accelerating voltage and Extended BSE Liberation Analysis measurement mode. MLA results were validated with ICP-OES chemical assays. More details on the flotation process and analytical procedures are available in Hoang et al. (2018). The data used in this case study is entirely available at Hoang et al. (2020).

The R package glmnet (Friedman et al., 2010) was used to train the lasso-regularized multinomial logistic regression (Figure $1 \mathrm{~B}$ ) and compute the cumulative recovery 
This is a peer-reviewed paper published in Minerals Engineering. Please submit any inquiries to the corresponding author. https://doi.org/10.1016/j.mineng.2021.107054

probability of each particle in the feed sample (Figure $1 \mathrm{C}$ ). The $\mathrm{R}$ base package stats (R Core Team, 2017) was used to fit the the classical first-order kinetic flotation model (Eq. (2), Sutherland, 1948) to each particle using non-linear least squares regression (Figure $1 \mathrm{D}$ ).

The weight distribution of the five flotation products can be found in the Supplementary Material 1, together with the overall number of particles in the corresponding MLA datasets, the class-label, and statistical weights used in the training phase of the particlebased separation model.

\subsection{Results}

\subsubsection{Particle-based separation models and validation}

Figure 2 displays boxplots comparing the predicted to the actual mass and mineralogical composition values of each concentrate and the final tailings using natural log ratios. The accessory mineral groups (oxides, sulfides, and micas) display the highest variability in the modelling results. Even though the median mass predicted for the first and fourth concentrate show a substantial deviation from their actual values (ca. 30\%), the tailings mass (and thus, its complementary, the total recovered mass) as well as most of the major components are correctly predicted, and the models are therefore considered satisfactory. Particularly, the results for apatite, the ore mineral, and carbonates, the most important gangue minerals, are excellent.

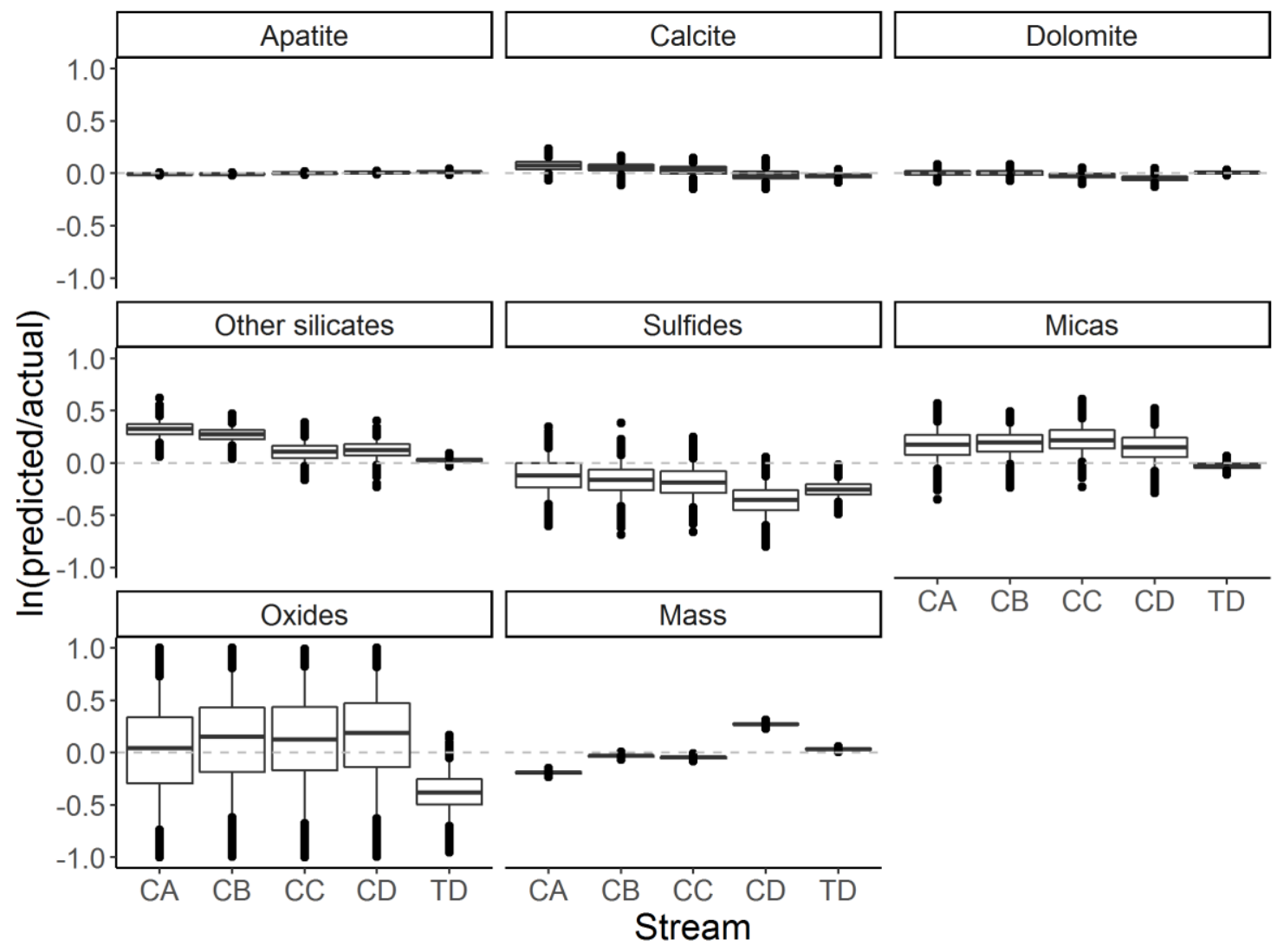

Figure 2: Boxplots comparing the predicted and actual flotation product's mass and composition using natural log ratios. Center lines represent the median, boxes represent the 0.25 and 0.75 percentiles, and whiskers the 0.05 and 0.95 percentiles. Dots represent the values beyond these thresholds, the outliers. 
This is a peer-reviewed paper published in Minerals Engineering. Please submit any inquiries to the corresponding author. https://doi.org/10.1016/j.mineng.2021.107054

\subsubsection{Flotation kinetics of single particles}

Figure 3 is used to evaluate the quality of the fit of a classical kinetic flotation model (cf. Section 2.3) for 49 randomly selected particles by comparing their computed recovery probability to the fit kinetic model. Figure 4 displays the goodness of fit of the classical first-order flotation model to each single particle in terms of $R^{2}$. The median $R^{2}$ is ca. 0.99 , clearly illustrating that the chosen model can successfully describe the recovery probability trend of each particle.

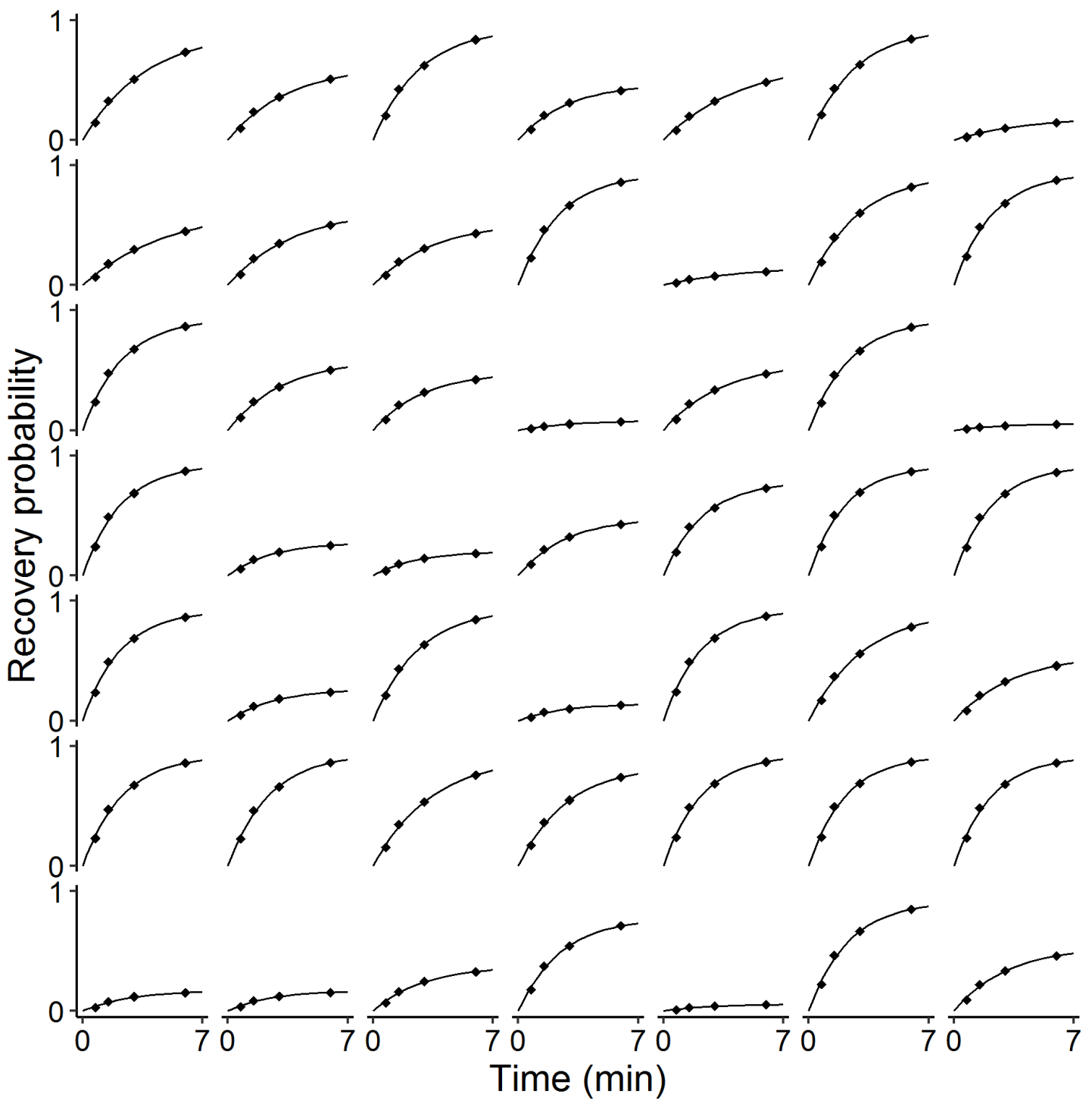

Figure 3: Comparison between calculated recovery probabilities (dots) and the classical kinetics flotation model (line) fit to 49 random particles. 
This is a peer-reviewed paper published in Minerals Engineering. Please submit any inquiries to the corresponding author. https://doi.org/10.1016/i.mineng.2021.107054

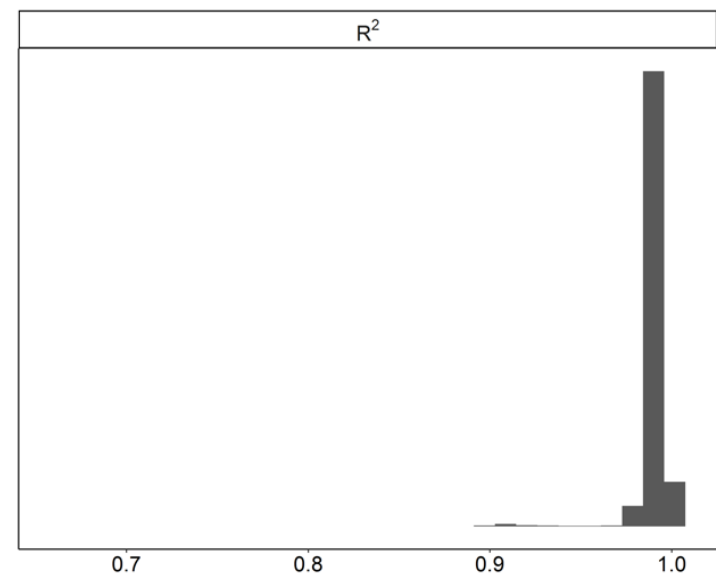

Figure 4: The goodness of fit of the classical first-order flotation model to each single particle as represented by $R^{2}$.

Figure 5 displays the distribution of $k_{m}$ for the different particles according to their main mineral component by mass. As expected, apatite-rich particles have a higher $k_{m}$ (i.e. better process performance) than the gangue-rich ones: the median $k_{m}$ of apatite-rich particles $\left(\sim 0.38 \mathrm{~min}^{-1}\right)$ is more than two times higher than the median $k_{m}$ of calcite- and dolomite-rich particles $\left(\sim 0.16 \mathrm{~min}^{-1}\right)$ and seven times higher than that of phlogopite and quartz $\left(<0.06 \mathrm{~min}^{-1}\right)$. These results also indicate a more efficient separation of apatite from silicates in comparison to carbonates. This is probably due to similarities in surface properties between the semi-soluble salt-type carbonate and calcium phosphate minerals (Hoang et al., 2018).

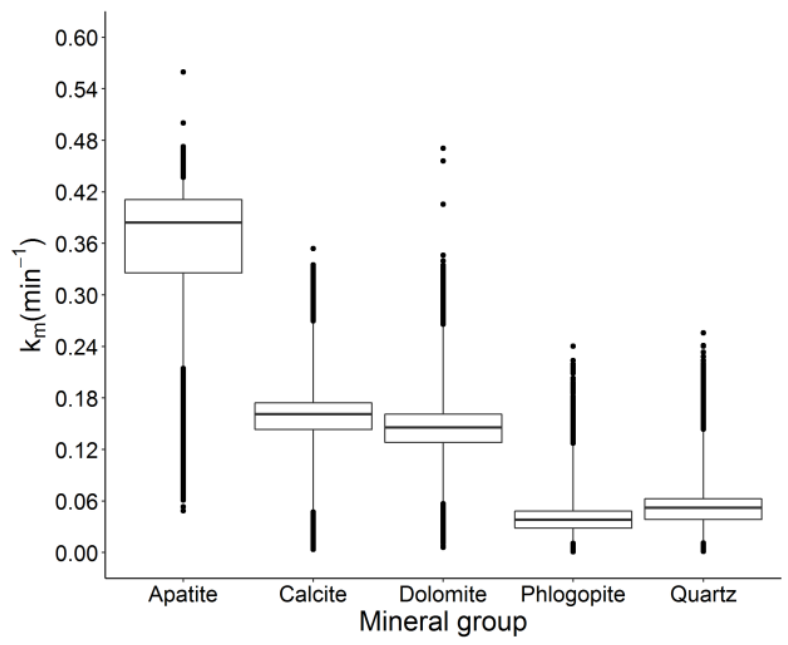

Figure 5: Distribution boxplots for $k_{m}$ of particles according to their main mineral component. Center lines represent the median, boxes represent the $25^{\text {th }}$ and $75^{\text {th }}$ percentiles, and whiskers the $1^{\text {st }}$ and $99^{\text {th }}$ percentiles. Dots represent the values beyond these thresholds as "outliers".

\subsubsection{Influence of particle properties on process behavior}

Given the number of variables $(70)$ and observations $(2,825,736)$ to be analyzed in this study, we provide an interactive platform in Pereira et al. (2020) that allows for entirely exploring the results obtained - the flotation behavior of more than five hundred thousand particles can be examined according to their intrinsic properties. Hence, we simplify the analysis of how particle properties influence process behavior by only considering the predominant minerals of the major mineral groups in the following paragraphs. 
This is a peer-reviewed paper published in Minerals Engineering. Please submit any inquiries to the corresponding author. https://doi.org/10.1016/j.mineng.2021.107054

When analyzing these results, one must bear in mind that the distribution of $k_{m}$-values is predominantly caused by particle properties. However, part of the observed variability derives from the probabilistic approach used in this method and stereological degradation.

Figure 6 displays the variation of $k_{m}$ values among particles with full surface liberation according to particle size and aspect ratio. The expected quasi-parabolic size influence on recovery (Wills and Finch, 2015) is clearly depicted for apatite, dolomite and calcite. Quartz and phlogopite particles are thus presumably recovered by entraintment only in this test. The carbonate minerals reach their maximum $k_{m}$ at a size of $\sim 10 \mu \mathrm{m}-$ a much finer size than apatite $(\sim 30 \mu \mathrm{m})$. In this study, we did not observe an effect of particle solidity on $k_{m}$.

Figure 7 displays the influence of particle size, liberation, aspect ratio, and mineralogy on the $k_{m}$ of particles containing apatite. Clearly, the poorly liberated apatite particles display a $k_{m}$ trend very similar to that of their main associated mineral, as depicted in Figure 6. Also, particles with surface liberation of apatite higher than $70 \%$ exhibit a similar $k_{m}$ trend to fully liberated apatite particles (Figure 6). Finally, particles in the intermediate liberation bin show a clear bimodal $k_{m}$ distribution. The influence of aspect ratio is less evident for non-liberated particles (Figure 7) than for the fully-liberated ones (Figure 6).

The recoverability of apatite particles with at least $70 \%$ free surface is very similar to that of the fully liberated particles, regardless of the associated minerals (Figures 6 and 7). Even though it translates into higher apatite recovery, this also poses a natural limitation to the highest obtainable concentrate grade, which could only be improved by regrinding.
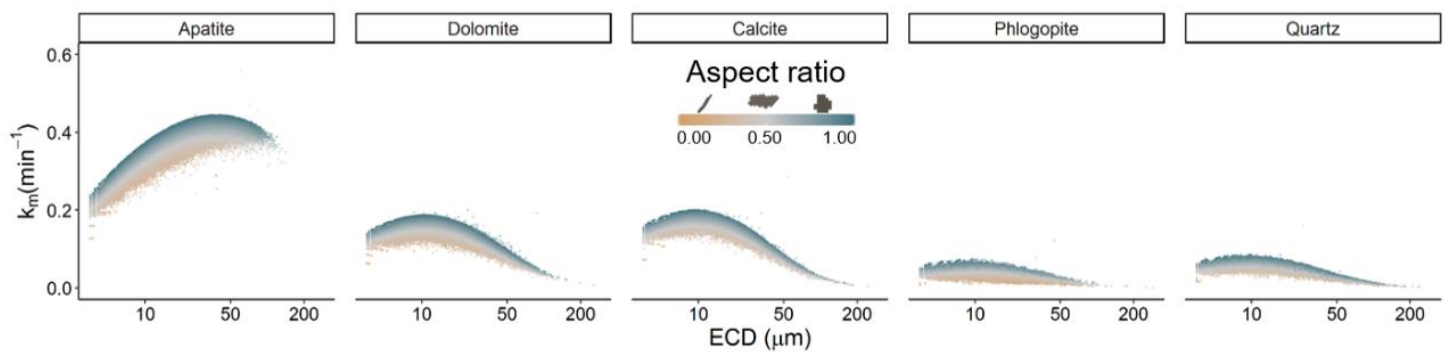

Figure 6: Distribution of $k_{m}$ among fully
size (equivalent circle diameter $-E C D$ ). 
This is a peer-reviewed paper published in Minerals Engineering. Please submit any inquiries to the corresponding author. https://doi.org/10.1016/i.mineng.2021.107054
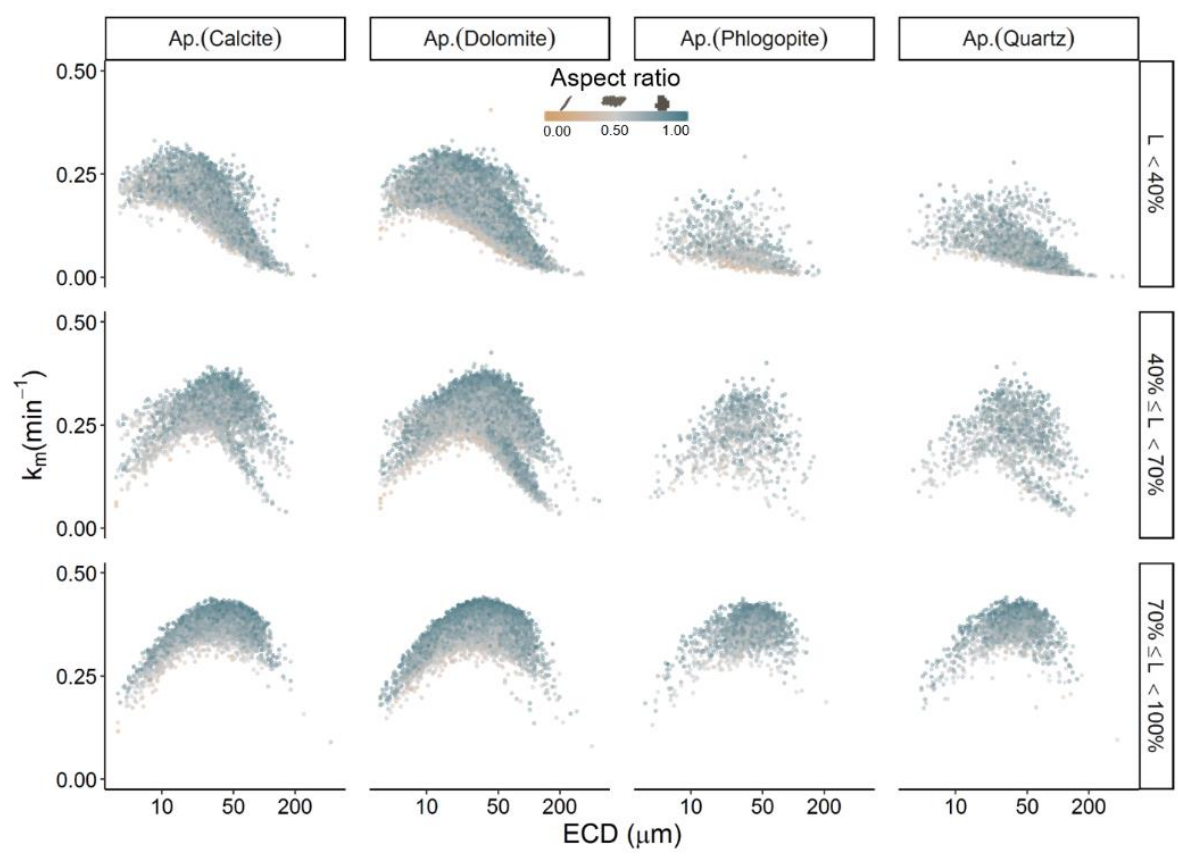

Figure 7: Distribution of $\mathrm{k}_{m}$ among particles containing apatite, according to their size (equivalent circle diameter - ECD), liberation degree ( $L$ ), and aspect ratio. Particles are further grouped according to their main association, as indicated in parentheses.

\section{Discussion and final thoughts}

We demonstrated above the level of detail attainable in the evaluation of kinetic flotation tests using our new particle-based method. In order to assess not only the correctness of these results, but to further highlight the advantages of this approach for data evaluation, we use the following paragraphs to compare its output with that of the more traditional approach used in the original study by Hoang et al. (2018).

First, we note that the new method is in excellent agreement with trends observed by Hoang et al. (2018). Specifically, apatite particles show the best flotation behavior as described by their $k_{m}$. The system is more selective in the removal of silicates than carbonates. The maximum $k_{m}$ for apatite dominated particles is attained at a size of $\sim 30 \mu \mathrm{m}$, and the $k_{m}$ values of phlogopite- and quartz-rich particles decrease with size, indicating that entrainment is possibly their major recovery mechanism. In addition, both approaches captured the well-described quasi-parabolic influence of size on the recoverability of apatite, dolomite, and calcite particles (Figure 6 ) by true flotation (Wills and Finch, 2015).

Second, with regard to the influence of particle properties, Figure 8 shows the recovery of particles containing apatite following the traditional particle-binning approach (A, Hoang et al., 2018), and cumulative recovery probability following the new particle-based method (B). Since particles are not binned in our approach (Figure $8 \mathrm{~B}$ ), the results display a much more detailed recovery probability distribution than would be possible with the traditional approach (Figure $8 \mathrm{~A}$ ).

For instance, we were able to jointly present the influence of shape, liberation and association on the recovery of apatite particles (Figure 7) and clearly recognize that nonliberated apatite particles have different flotation behavior according to their associated mineral. These findings expand the outcomes of many flotation studies that focused solely on the influence of surface liberation to explain the recovery of a particle (Gorain 
This is a peer-reviewed paper published in Minerals Engineering. Please submit any inquiries to the corresponding author. https://doi.org/10.1016/j.mineng.2021.107054

et al., 2000; Hoang et al., 2018; Jameson, 2012; Lamberg and Vianna, 2007; Lotter et al., 2011).

A

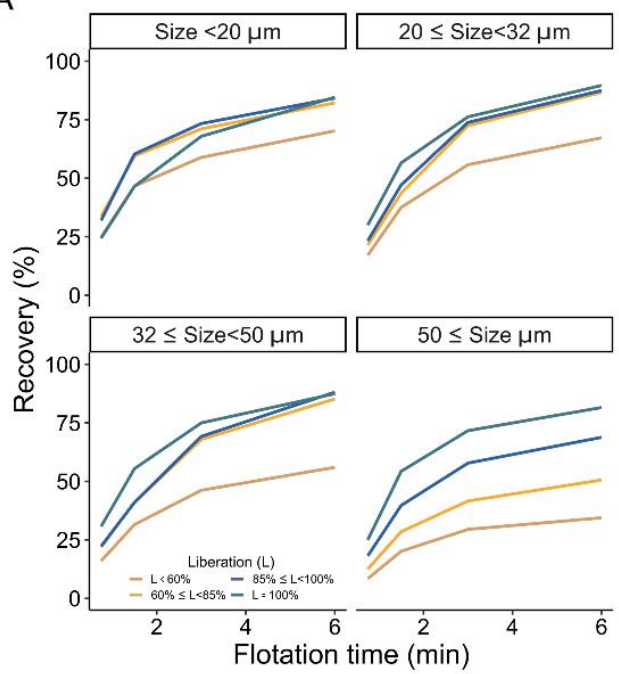

B

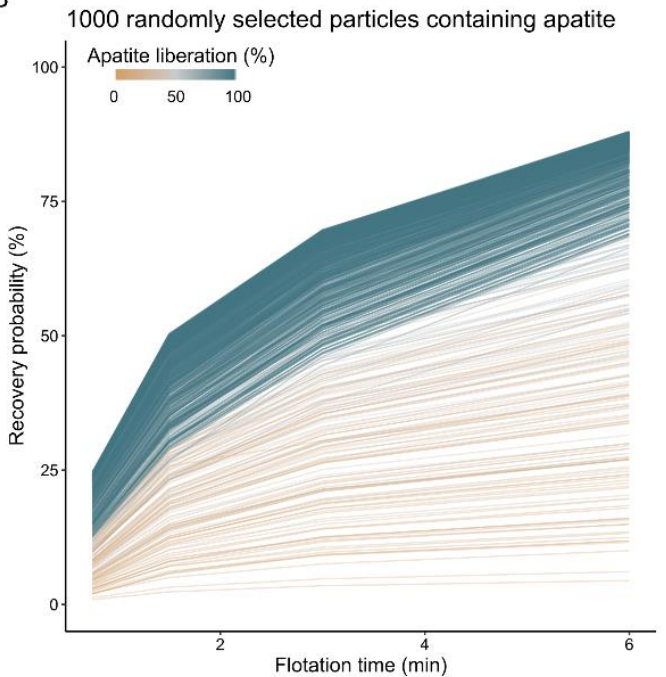

Figure 8: Recovery of particles containing apatite illustrated by the traditional particle binning method (A, Hoang et al., 2018) and the novel particle based approach (B). The liberation depicted in $B$ is based on freesurface.

Our results contradict the contention that non-spherical particles will float more easily than their spherical counterparts (Verrelli et al., 2014; Vizcarra et al., 2011). Rather, our results suggest that particles of higher aspect ratio, i.e. the least elongated or flat ones (Pourghahramani and Forssberg, 2005), have a higher chance of being recovered in the concentrate fraction irrespective of their constituting mineral, size and liberation (Figure 6). Our findings are in agreement with the contributions of Schmidt and Berg (1997, 1996) and Little et al. (2016). This influence might be attributed to the stability of coalescence, a combined effect of the particle-bubble collision hydrodynamics, the froth lamella drainage behavior, and the drop-back of previously attached particles from the froth. More test work is required to investigate this hypothesis.

Furthermore, we do not observe a significant effect of solidity (Pereira et al., 2020) on the flotation behavior of particles in our case study. This could either mean that particle solidity does not influence recovery, or that the Mineral Liberation Analyzer could not capture this property in sufficient detail in our case study. We recommend the future use of 3D mineral characterization systems, such as X-ray computed tomography (Ketcham and Carlson, 2001), albeit with reservations about its potential resolution, for better investigating the influence of shape properties in the recovery of particles by flotation. Similarly to Pereira et al. (2021), our approach can promptly work with particle descriptive datasets from different characterization systems.

Third, our method minimizes bin representativity issues commonly present in the particle binning approach. As discussed by Hoang et al. (2018), some incongruent results arise from the low representativity of particles in some size/liberation bins. For example, fully liberated apatite particles show the highest recovery in comparison to other liberation

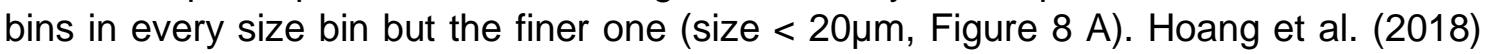
attributed this divergence to the agglomeration of fine particles during sample preparation, which unbalanced the fine particle distribution among the different liberation bins. However, if critical agglomeration problems were present in the data, our method would have failed to capture the influence of surface liberation on particle recovery 
This is a peer-reviewed paper published in Minerals Engineering. Please submit any inquiries to the corresponding author. https://doi.org/10.1016/j.mineng.2021.107054

(Figure $8 \mathrm{~B}$ clearly illustrates that this is not the case). The multinomial logistic regression minimizes issues with bin representativity by capturing the influence of each particle property on its process behavior, continuously, over the entire property distribution space.

Nevertheless, one can observe in Figure 7 a distribution along two trends for the intermediate apatite liberation bin $(40 \% \leq$ Liberation $<70 \%)$ irrespective of its association bin, which indicates some granularity or discontinuity of the model. Recalling the model structure presented in Section 2.2, we add an interaction between shape and size descriptive variables and a categorical variable indicating the main mineral in mass of each particle. This strategy allows us to capture a mineral-specific effect of particle dimension properties on its recoverability. However, it does not constitute a continuous representation of variations in particle composition. Thus different particle size effects are fit for particles that have been grouped into the same liberation and association bins in Figure 7, but have different main minerals in mass. On the one hand, this leads to the bimodal distribution observed. On the other hand, it reinforces the observation that the influence of particle size varies with particle mineralogy, similar to what is observed for fully-liberated particles in Figure 6. Future studies should evaluate other strategies to incorporate variable interactions, taking into consideration the limitations of the (multinomial) logistic regression.

Additionally, the variable interaction structure discussed above allows for incorporating additional information into our particle-based kinetic flotation models. Vos (2017) demonstrated the potential influence particle surface structures on the flotation response of particles. Yet, this author could not fully incorporate this effect into their kinetic flotation models due to the lack of a robust modelling approach for composite particles. Using the method presented in this manuscript, particle surface structure be introduced into the particle dataset as a categorical variable and thus used for the kinetic flotation modelling.

Fourth, we showed that the classical kinetic flotation model successfully describes the particle recovery probability trends for the experiment studied in this manuscript. This confirms our theoretical expectation. Since most of the complexity present in other kinetic flotation models is due to variations in the assumed distributions of particle properties (Polat and Chander, 2000), these models are not relevant for cases in which the behavior of single particles is concerned. Therefore, we recommend that the classic flotation model should also be used in future applications of our method.

Fifth, similar to the method presented by Pereira et al. (2021), the approach introduced in this manuscript is not limited to flotation. It can be applied to any (raw material) separation process, independent of the number of output streams, making it a versatile tool in the study of mineral separation circuits.

Finally, we note that this study was limited to the consideration of a single experimental setup. Subsequent studies should explore its potential to compare process outcomes under different experimental setups. This could be used to identify optimum operating conditions and to understand the influence of machine/process parameters on particle recovery.

We anticipate that our method will assist further developments in the field of mineral beneficiation, especially flotation, given the resolution at which it allows separation processes to be observed and analyzed. After the acquisition of particle data, the method requires only a few minutes to train the predictive models on a conventional computer, and its output results can be swiftly forwarded to interactive platforms (Pereira et al., 
This is a peer-reviewed paper published in Minerals Engineering. Please submit any inquiries to the corresponding author. https://doi.org/10.1016/j.mineng.2021.107054

2020), which ease its use in routine laboratory or plant surveys. Moreover, the method is self-adaptive and has a good forecasting potential, which makes it useful for the optimization of minerals processing operations.

\section{Acknowledgements}

The authors thank the German Federal Ministry for Education and Research (BMBF) within the CLIENT-II initiative for funding this study (Grant number 033R189B). We thank our colleagues Markus Buchmann, Edgar Schach, Marius Kern, and Nathalie Kupka for fruitful discussions during method development, and Peter Seidel, Alejandro Abadias, and two anonymous reviewers for comments on the text.

\section{Credit author contributions}

Lucas Pereira: Conceptualization, Methodology, Software, Visualization, Writing Original Draft. Max Frenzel: Conceptualization, Methodology, Supervision, Writing Original Draft. Duong Huu Hoang: Investigation, Writing - Review \& Editing. Raimon Tolosana-Delgado: Methodology, Software, Writing - Review \& Editing. Martin Rudolph: Investigation, Writing - Review \& Editing. Jens Gutzmer: Supervision, Writing - Review \& Editing.

\section{References}

Albijanic, B., Ozdemir, O., Nguyen, A. V., Bradshaw, D., 2010. A review of induction and attachment times of wetting thin films between air bubbles and particles and its relevance in the separation of particles by flotation. Adv. Colloid Interface Sci. 159, 1-21. https://doi.org/10.1016/j.cis.2010.04.003

Bish, D.L., Post, J.E., 1989. Modern Powder Diffraction, Volume 20. ed, Reviews in Mineralogy volume 20. Mineralogical society of America, Chantilly, VA.

Do, H., 2010. Development of a Turbulent Flotation Model from First Principles. Virginia Polytechnic Institute and State University. https://doi.org/10.1017/CBO9781107415324.004

Dobby, G.S., Savassi, O.N., 2005. An advanced modelling technique for scale-up of batch flotation results to plant metallurgical performance. Australas. Inst. Min. Metall. Publ. Ser. 99-103.

Dowling, E.C., Klimpel, R.R., Aplan, F.F., 1985. Model Discrimination in the Flotation of a Porphyry Copper Ore. Trans. Am. Inst. Mining, Metall. Pet. Eng. Soc. 278, 87102. https://doi.org/10.1007/bf03402602

Fandrich, R., Gu, Y., Burrows, D., Moeller, K., 2007. Modern SEM-based mineral liberation analysis. Int. J. Miner. Process. 84, 310-320. https://doi.org/10.1016/j.minpro.2006.07.018

Frenzel, M., Bachmann, K., Carvalho, J.R.S., Relvas, J.M.R.S., Pacheco, N., Gutzmer, J., 2019. The geometallurgical assessment of by-products-geochemical proxies for the complex mineralogical deportment of indium at Neves-Corvo, Portugal. Miner. Depos. 54, 959-982. https://doi.org/10.1007/s00126-018-0849-6

Friedman, J., Hastie, T., Tibshirani, R., 2010. Regularization Paths for Generalized Linear Models via Coordinate Descent. J. Stat. Software, Artic. 33, 1-22. https://doi.org/10.18637/jss.v033.i01

Gharai, M., Venugopal, R., 2015. Modeling of flotation process - an overview of different approaches. Miner. Process. Extr. Metall. Rev. 37, 08827508.2015.1115991. https://doi.org/10.1080/08827508.2015.1115991 
This is a peer-reviewed paper published in Minerals Engineering. Please submit any inquiries to the corresponding author. https://doi.org/10.1016/j.mineng.2021.107054

Gorain, B.K., Franzidis, J.P., Ward, K., Johnson, N.W., Manlapig, E. V., 2000. Modeling of the Mount Isa rougherscavenger copper flotation circuit using size-by-liberation data. Miner. Metall. Process. 17, 173-180. https://doi.org/10.1007/bf03402844

Gu, Y., Schouwstra, R.P., Rule, C., 2014. The value of automated mineralogy. Miner. Eng. 58, 100-103. https://doi.org/10.1016/j.mineng.2014.01.020

Guilbert, J.M., Park, C.F., 2007. The Geology of Ore Deposits. Waveland Press.

Hastie, T., Tibshirani, R., Wainwright, M., 2015. Statistical Learning with Sparsity, Statistical Learning with Sparsity: The Lasso and Generalizations. Chapman and Hall/CRC. https://doi.org/10.1201/b18401

Heinig, T., Bachmann, K., Tolosana-Delgado, R., Boogaart, G. Van Den, Gutzmer, J., 2015. Monitoring gravitational and particle shape settling effects on MLA sampling preparation, in: IAMG Conference 2015. pp. 200-206.

Henderson, A.R., 2005. The bootstrap: A technique for data-driven statistics. Using computer-intensive analyses to explore experimental data. Clin. Chim. Acta 359, 126. https://doi.org/10.1016/j.cccn.2005.04.002

Hoal, K.O., 2008. Getting the geo into geomet. SEG Newsletter, Soc. Econ. Geol. 73, $11-15$.

Hoang, D.H., Kupka, N., Peuker, U.A., Rudolph, M., 2018. Flotation study of fine grained carbonaceous sedimentary apatite ore - Challenges in process mineralogy and impact of hydrodynamics. Miner. Eng. 121, 196-204. https://doi.org/10.1016/j.mineng.2018.03.021

Hoang, D.H., Pereira, L., Kupka, N., Tolosana-Delgado, R., Frenzel, M., Rudolph, M., Gutzmer, J., 2020. Automated mineralogy particle dataset: apatite flotation [Data set]. https://doi.org/doi.org/10.14278/rodare.542

Jameson, G.J., 2012. The effect of surface liberation and particle size on flotation rate constants. Miner. Eng. 36-38, 132-137. https://doi.org/10.1016/j.mineng.2012.03.011

Jenkins, R., 2008. X-Ray Fluorescence Spectrometry, Handbook of Analytical Techniques. https://doi.org/10.1002/9783527618323.ch23

Keppel, G., Wickens, T.D., 2004. Design and Analysis: A Researcher's Handbook. Prentice Hall.

Kern, M., Möckel, R., Krause, J., Teichmann, J., Gutzmer, J., 2018. Calculating the deportment of a fine-grained and compositionally complex Sn skarn with a modified approach for automated mineralogy. Miner. Eng. 116, 213-225. https://doi.org/10.1016/j.mineng.2017.06.006

Ketcham, R. a., Carlson, W.D., 2001. Acquisition, optimization and interpretation of Xray computed tomographic imagery: applications to the geosciences. Comput. Geosci. 27, 381-400. https://doi.org/10.1016/S0098-3004(00)00116-3

King, R.P., Schneider, C.L., King, E.A., 2012. Modeling and Simulation of Mineral Processing Systems, Second. ed. Society for Mining, Metallurgy, and Exploration, Englewood, California.

Kupka, N., Tolosana-Delgado, R., Schach, E., Bachmann, K., Heinig, T., Rudolph, M., 2020. $R$ as an environment for data mining of process mineralogy data: A case study of an industrial rougher flotation bank. Miner. Eng. 146. https://doi.org/10.1016/j.mineng.2019.106111

Lamberg, P., Vianna, S., 2007. A technique for tracking multiphase mineral particles in flotation circuits. XXII Encontro Nac. Trat. Minérios e Metal. Extrativa - VII Meet. South. Hemisph. Miner. Technol. 195-202. 
This is a peer-reviewed paper published in Minerals Engineering. Please submit any inquiries to the corresponding author. https://doi.org/10.1016/j.mineng.2021.107054

Leißner, T., Hoang, D.H.H., Rudolph, M., Heinig, T., Bachmann, K., Gutzmer, J., Schubert, H., Peuker, U.A. a., 2016. A mineral liberation study of grain boundary fracture based on measurements of the surface exposure after milling. Int. J. Miner. Process. 156, 3-13. https://doi.org/10.1016/j.minpro.2016.08.014

Little, L., Wiese, J., Becker, M., Mainza, A., Ross, V., 2016. Investigating the effects of particle shape on chromite entrainment at a platinum concentrator. Miner. Eng. 9697, 46-52. https://doi.org/10.1016/j.mineng.2016.06.003

Lotter, N.O., Kormos, L.J., Oliveira, J., Fragomeni, D., Whiteman, E., 2011. Modern process mineralogy: Two case studies. Miner. Eng. 24, 638-650. https://doi.org/10.1016/j.mineng.2011.02.017

Miller, J.D., Lin, C.-L., Hupka, L., Al-Wakeel, M.I., 2009. Liberation-limited grade/recovery curves from X-ray micro CT analysis of feed material for the evaluation of separation efficiency. Int. J. Miner. Process. 93, 48-53. https://doi.org/10.1016/j.minpro.2009.05.009

Pawlowsky-Glahn, V., Egozcue, J.J., Tolosana-Delgado, R., 2015. Modeling and Analysis of Compositional Data, Statistics in Practice. Wiley.

Pereira, L., Frenzel, M., Hoang, D.H., Tolosana-Delgado, R., Rudolph, M., Gutzmer, J., 2020. Interactive results viewer: Computing single-particle flotation kinetics using automated mineralogy data and machine learning. https://doi.org/doi.org/10.14278/rodare.535

Pereira, L., Frenzel, M., Khodadadzadeh, M., Tolosana-Delgado, R., Gutzmer, J., 2021. A self-adaptive particle-tracking method for minerals processing. J. Clean. Prod. 279, 123711. https://doi.org/10.1016/j.jclepro.2020.123711

Polat, M., Chander, S., 2000. First-order flotation kinetics models and methods for estimation of the true distribution of flotation rate constants. Int. J. Miner. Process. 58, 145-166. https://doi.org/10.1016/S0301-7516(99)00069-1

Pourghahramani, P., Forssberg, E., 2005. Review of applied particle shape descriptors and produced particle shapes in grinding environments. Part I: particle shape descriptors. Miner. Process. Extr. Metall. Rev. 26, 145-166. https://doi.org/10.1080/08827500590912095

R Core Team, 2017. R: A Language and Environment for Statistical Computing.

Runge, K., 2010. Laboratory flotation testing--an essential tool for ore characterisation, in: Flotation Plant Optimisation: A Metallurgical Guide to Identifying and Solving Problems in Flotation Plants. Australasian Institute of Mining and Metallurgy, Spectrum Series Carlton, Vic, pp. 55-173.

Saerens, M., Latinne, P., Decaestecker, C., 2002. Adjusting the Outputs of a Classifier to New a Priori Probabilities: A Simple Procedure. Neural Comput. 14, 21-41. https://doi.org/10.1162/089976602753284446

Sandmann, D., 2015. Method Development in Automated Mineralogy. TU Bergakademie Freiberg.

Schmidt, D.C., Berg, J.C., 1997. A Preliminary Hydrodynamic Analysis of the Flotation of Disk-Shaped Toner Particles. Prog. Pap. Recycl. 6, 38-49.

Schmidt, D.C., Berg, J.C., 1996. The effect of particle shape on the flotation of toner particles. Prog. Pap. Recycl. 5, 67-77.

Sutherland, K.L., 1948. Physical chemistry of flotation. XI: Kinetics of the flotation process. J. Phys. Colloid Chem. 52, 394-425. https://doi.org/10.1021/j150458a013

Verrelli, D.I., Bruckard, W.J., Koh, P.T.L., Schwarz, M.P., Follink, B., 2014. Particle shape effects in flotation. Part 1: Microscale experimental observations. Miner. Eng. 
This is a peer-reviewed paper published in Minerals Engineering. Please submit any inquiries to the corresponding author. https://doi.org/10.1016/j.mineng.2021.107054

58, 80-89. https://doi.org/10.1016/j.mineng.2014.01.004

Vizcarra, T.G., Harmer, S.L., Wightman, E.M., Johnson, N.W., Manlapig, E.V., 2011. The influence of particle shape properties and associated surface chemistry on the flotation kinetics of chalcopyrite. Miner. Eng. 24, 807-816. https://doi.org/10.1016/j.mineng.2011.02.019

Vos, F., 2017. The effect of mineral grain textures at particle surfaces on flotation response. The University of Queensland. https://doi.org/10.14264/uql.2017.326

Wang, L., Peng, Y., Runge, K., Bradshaw, D., 2015. A review of entrainment: Mechanisms, contributing factors and modelling in flotation. Miner. Eng. 70, 77-91. https://doi.org/10.1016/j.mineng.2014.09.003

Wills, B.A., Finch, J., 2015. Wills' Mineral Processing Technology: An Introduction to the Practical Aspects of Ore Treatment and Mineral Recovery. Elsevier Science.

Xu, M., 1998. Modified flotation rate constant and selectivity index. Miner. Eng. 11, 271278. https://doi.org/10.1016/S0892-6875(98)00005-3

Yoon, R.-H., Mao, L., 1996. Application of Extended DLVO Theory, IV. J. Colloid Interface Sci. 181, 613-626. https://doi.org/10.1006/jcis.1996.0419

Yoon, R.H., Kelley, K., Do, H., Sherell, I., Noble, A., Kelles, S., Soni, G., 2012. Development of a flotation simulator based on a first principles model, in: 26th International Mineral Processing Congress, IMPC. pp. 5969-5982. 